\title{
Alternative selection of processing additives to enhance the lifetime of OPVs
}

J. Kettle ${ }^{a^{*}}$, H. Waters ${ }^{a}$, M. Horie ${ }^{b}$, G.C. Smith ${ }^{c}$

a School of Electronic Engineering, University of Bangor, Dean Street, Bangor LL57 1UT, Wales, UK

${ }^{b}$ Department of Chemical Engineering, National Tsing Hua University, 101, Section 2, Kuang-Fu Road, Hsinchu 30013, Taiwan ROC

c Department of Natural Sciences, University of Chester, Thornton Science Park, Chester CH2 4NU, UK

\section{Abstract}

The use of processing additives is known to accelerate the degradation of Organic Photovoltaics (OPVs) and therefore, this paper studies the impact of selecting alternative processing additives for PCPDTBT:PC ${ }_{71} B M$ solar cells in order to improve the stability. The use of naphthalene-based processing additives has been undertaken, which is shown to reduce the initial power conversion efficiency by $23 \%-42 \%$, primarily due to a decrease in the short-circuit current density, but also fill factor. However, the stability is greatly enhanced by using such additives, with the long term stability $\left(T_{50 \%}\right)$ enhanced by a factor of four. The results show that there is a trade-off between initial performance and stability to consider when selecting the initial process additives. XPS studies have provided some insight into the decreased degradation and show that using 1-chloronaphthalene (CIN) leads to reduced morphology changes and reduced oxidation of the thiophene-ring within the PCPDTBT backbone.

\section{Introduction}

Organic Photovoltaics (OPVs) provide the possibility of low cost, solution processible solar electricity generation. Whilst performances have steadily improved over the past 10 years, OPVs still exhibit poor lifetimes. This is exasperated by the use of 'processing additives;' as shown in two recent reports of the stability of solar cells made with Poly(\{4,8-bis[(2-ethylhexyl)oxy]benzo[1,2-b:4,5$b^{\prime}$ ]dithiophene-2,6-diyl\}\{3-fluoro-2-[(2-ethylhexyl)carbonyl]thieno[3,4-b]thiophenediyl\}) (abbrev.: PTB7) and poly[(4,4-bis(2-ethylhexyl)-cyclopenta-[2,1-b;3,4-b']dithiophene)-2,6-diyl-alt-2,1,3benzothiadiazole-4,7-diyl] (abbrev.: PCPDTBT) as well as other materials [1-3]. Processing additives 
based on alkanethiols, halogenated naphthalenes or similar co-solvents are used in almost all reports of high efficiency OPVs and acts as a non-reacting solvent [4-5]. It has been shown that the incorporation of a few volume percent of 1,8-octanedithiol (ODT) into the blend improves the Power Conversion Efficiency (PCE) by about a factor of two. It does this by modifying the morphology and controlling the phase separation between the donor and acceptor components [6]. Reports indicate that $\mathrm{ODT}$ leads to enhanced connectivity of $\mathrm{PC}_{71} \mathrm{BM}$ networks [7], a small increase in polymer crystallinity [8], reduced charge recombination and reduced charge carrier loss [9]. However, recent data indicates that inclusion of additives has a detrimental effect on the stability of the solar cell. Under stability measurements, solar cell devices made with processing additives tend to degrade quicker than devices made without additives. The likely reason for this is related to the high boiling point of the processing additive; typically greater than $150^{\circ} \mathrm{C}$. From the analysis of active layer material systems, it is assumed that the processing additive remains in the film after processing, or creates an initially unstable morphology, and continues to detrimentally alter the active layer morphology.

In this paper the use of alternative processing additives is reported to identify if efficiency can be increased without compromising device lifetime. In particular, the work is targeted at lower boiling-point additives, as these are more likely to evaporate than alkanethiols. OPVs are fabricated with a range of additives and tested for efficiency and lifetime. In addition, the material changes are studied using X-ray Photoelectron Spectroscopy (XPS)

\section{Experimental}

\subsection{Device fabrication}

Initially, indium tin oxide (ITO) coated glass substrates $\left(R_{s}=16 \Omega /\right.$ square) were cleaned using solvents. Subsequently, a 40nm PEDOT:PSS layer was deposited using spin coating and dried at $120^{\circ} \mathrm{C}$ for 30 minutes. PCPDTBT was synthesised in-house and possesses 2-ethylhexyl solubilising side chains [10]. Three blends were prepared whereby the polymer was mixed with a fullerene derivative. The PCPDTBT and $\mathrm{PC}_{71} \mathrm{BM}$ blends were mixed with weight ratios 1:3 with chlorobenzene solvent using a concentration of $30 \mathrm{mg} / \mathrm{mL}$. Three different processing additives were trialled for this work; ODT, both 1-bromonaphthalene $(\mathrm{BrN})$ and 1-chloronaphthalene $(\mathrm{CIN}) \mathrm{w} / \mathrm{w}$ of $2.5 \%$. The additives, $\mathrm{BrN}$ and $\mathrm{CIN}$, were selected due to their low boiling points of $119^{\circ} \mathrm{C}$ and $139^{\circ} \mathrm{C}$, respectively and effectiveness when applied to blends of other active layer materials such as P3HT:PCBM [10]. For all blends, the same batch of PCPDTBT and PC71BM were used, so the data 
obtained compares only the changes in process additives. The parameters such as film thickness (as a function of spin-casting speed) and annealing temperatures were optimised for each blend. Prior to coating, all blends were allowed to dissolve for 24 hours on a hot plate stirrer and filtered using a $0.45 \mu \mathrm{m}$ PTFE filter. All coatings were undertaken in a glovebox and annealed to remove any residual solvent; a procedure optimised for device fabrication [10]. Finally, the cathode electrode was deposited, which consisted of $8 \mathrm{~nm}$ calcium ( $\mathrm{Ca}$ ) followed by $100 \mathrm{~nm}$ of Aluminium (Al), to define an active area size of $0.5 \mathrm{~cm}^{2}$. A schematic of the device setup is shown in Figure 1.

Devices were tested for current density-Voltage (J-V) using a Newport solar simulator with $100 \mathrm{mWcm}^{-2}$ AM1.5G output (calibrated using a silicon reference cell from RERA in the Netherlands). For ageing of devices, light soaking was conducted using a halogen light soaking system. Devices were kept at open circuit in between measurements and J-V measurements were made every 30 minutes. This was conducted in accordance with ISOS-L-2 standards [11].

\subsection{XPS experiments}

The XPS data were acquired using a bespoke ultra-high vacuum system fitted with a Specs GmbH Focus 500 monochromated Al Ka X-ray source, Specs GmbH Phoibos $150 \mathrm{~mm}$ mean radius hemispherical analyser with 9-channeltron detection, and a Specs GmbH FG20 charge neutralising electron gun. Survey spectra were acquired using Al Ka radiation at $1486.6 \mathrm{eV}$ over the binding energy range $1100-0 \mathrm{eV}$ using a pass energy of $50 \mathrm{eV}$, and high resolution scans were made over the $C 1 s, 0$ 1s and $S 2 p$ lines using a pass energy of $15 \mathrm{eV}$. In each case, the analysis was an areaaverage over a region approximately $2 \mathrm{~mm}$ in diameter on the sample surface. The energy scale of the instrument is calibrated according to ISO standard 15472, and the intensity scale is calibrated using an in-house method traceable to the UK National Physical Laboratory.

\section{Results}

\subsection{Solar cell performance}

Initially, optimising of the process conditions for OPVs with different additives was conducted. The optimum active layer thickness was found to be $160 \mathrm{~nm}$ and $155 \mathrm{~nm}$ for $\mathrm{CIN}$ and $\mathrm{BrN}$, respectively. This compares closely to the optimal thickness for OPVS made with 1,8-octanedithiol (ODT) additive which was $140 \mathrm{~nm}$. In addition to optimising the spin-speed, annealing temperature and drying conditions were also investigated. After extensive optimisation in other papers [12,13], many groups have shown that PCPDTBT:PC ${ }_{71} \mathrm{BM}$ including ODT additive operate best with $80^{\circ} \mathrm{C}$ annealing. This 
was also found to be the optimum temperature for active layer annealing when using $\mathrm{BrN}$ and $\mathrm{CIN}$ additives also. Annealing was trialled at higher temperatures, but led to a decrease in Power Conversion Efficiency (PCE). For example, annealing at $140^{\circ} \mathrm{C}$ led to a relative decrease in PCE of $20 \%$, as a result of decrease of in all performance parameters, namely short-circuit current density $\left(\mathrm{J}_{\mathrm{SC}}\right)$, Fill Factor (FF) and open-Circuit Voltage $\left(\mathrm{V}_{\mathrm{OC}}\right)$.

A summary of the optimum performance of PCPDTBT:PC ${ }_{71} \mathrm{BM}$ OPVs manufactured with each processing additive and measured under AM1.5G conditions is shown in Table 1, with the J-V characteristics shown in Figure 2. It is clear that using the conventional additive, ODT, leads to the best PCE. As can be seen with data in Table 1, the difference in PCE between additives is primarily due to lower FF and $\mathrm{J}_{\mathrm{SC}}$, suggesting large morphological variance between the active layers and a reduced photo-generation in active layers containing $\mathrm{BrN}$ and $\mathrm{CIN}$ solvents. Interestingly, a correlation exists between boiling point of the processing additive and final device performance; $\mathrm{BrN}$ has the lowest boiling point and also the lowest PCE, whereas ODT possesses the highest boiling point and PCE. As OPV cells made with ODT possessed a higher $\mathrm{J}_{s C}$ and FF than those that include CIN or $\mathrm{BrN}$, it can therefore be assumed that $\mathrm{BrN}$ led to the least optimal morphology, due to its lower boiling temperature. It is worth noting that the performances of these cells are slightly lower than the previous devices from the group due to the larger active area sizes used for these tests. AFM analyses were conducted on the surfaces of PCPDTBT.PC71BM based films, which had been cast with $\mathrm{CIN}, \mathrm{BrN}$ or ODT. Figure 3 shows topographical images of these films for scan areas of $5 \mu \mathrm{m} x$ $5 \mu \mathrm{m}$. It can be seen from Figure 3 (a) that films made with $\mathrm{BrN}$ exhibit the greatest surface roughness and largest aggregates, whereas a much smoother film and lower aggregation is observed with the films processed with the ODT additive. This would explain the lower PCE of cells made with $\mathrm{BrN}$; it is evident that the use of this additive leads to a worse initial morphology as such large aggregates are shown to reduce significantly performance [1], which explains the lower initial PCE of these cells.

\subsection{Solar cell lifetime}

In addition to optimising fabrication conditions, OPV cells of PCPDTBT.PC71BM with ODT, CIN or BrN were tested for stability. Figure Figure 4 shows how the $V_{O C}, J_{S C}, F F$ and PCE changed with time for cells made using ODT, CIN or BrN. It appears that layers made with BrN leads to the greatest stability. Unfortunately, this also showed the lowest initial PCE (see table 1). A good measure of the stability is to consider the time taken for the device to fall to $50 \%$ of the original value ( $\left.T_{50 \%}\right)$. The $T_{50 \%}$ 
for the best performing cells made with $\mathrm{CIN}$ and $\mathrm{BrN}$ was measured to be 90 hours and 115 hours. Cells made with ODT possess $\mathrm{T}_{50 \%}$ of 25 hours. Interestingly, the data for improved $\mathrm{T}_{50 \%}$ appears to be correlated to lower additive boiling temperature.

From the data in figure 4 and Table 1, it is evident that the cells made with ODT possess substantially worse stability. Although ODT shows reduced stability, its initial PCE is at least twice that of cells using either $\mathrm{CIN}$ or BrN. Therefore, when comparing the relative performance of an OPV over its lifetime, a better indicator is the total power generated over the devices' lifetimes. This takes into account both the different initial PCE as well as the longer term stability of the device. The cell lifetime is defined as between the period of 0 hours until $\mathrm{T}_{10 \%}$ is reached and this data is shown in Table 2. From the data in Table 2, it is observed that devices made with $\mathrm{CIN}$ exhibit the best power generating capabilities, with greater than 2-fold increase in power generation observed over the lifetime when compared to a device made with ODT. Whilst devices made with $\mathrm{BrN}$ have a greater stability and those made with ODT possess higher PCE, the use of CIN appears to give the best tradeoff between stability and performance.

Previous work in the area has supposed that the high boiling point of processing additive is likely to lead to the additive remaining in the active layer after processing, or leads to an unstable initial morphology [1]. This residual solvent/unstable morphology continues to dynamically alter the morphology with time, leading to enhanced degradation. The data in this section indicates that by using a more volatile additive, which evaporates quicker, the morphological changes that occur from the dynamically altering of the active layer can be reduced, enhancing the stability. Whilst the data is not shown, the authors found that the most stable devices for all additives was obtained by annealing at $80^{\circ} \mathrm{C}$. This is an interesting conclusion and shows that 'over-annealing' at higher temperatures (up to $140^{\circ} \mathrm{C}$ was trialled) to remove as much of the additive as possible in the active layer doesn't necessarily lead to improved stability. The likely cause of this is that the higher temperature annealing might instigate a new degradation mechanism such as a less stable morphology. However, the work cannot rule out the possibility that ODT could react with the calcium interface layer, which could also account for the increased degradation rate.

\subsection{Analysis of morphological and chemical changes using XPS measurements}

Data was collated from the active layers of PCPDTBT-based solar cells using XPS to better understand

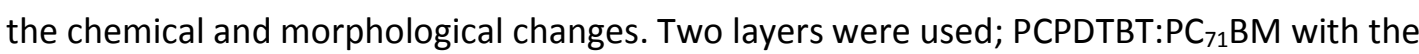
processing additive, $\mathrm{ODT}$, and PCPDTBT:PC ${ }_{71} \mathrm{BM}$ with the $\mathrm{CIN}$. Active layers made with $\mathrm{BrN}$ were 
excluded from this study as the CIN showed the most promise. All blends were prepared with $75 \%$ fullerene content, in keeping with the optimal performance in device active layers and applied onto PEDOT-coated glass substrates. As both films contain a $75 \%$ fullerene content, we can expect the molecular structures of PCPDTBT:PC ${ }_{71} \mathrm{BM}$ to possess a stoichiometry of $96.1 \% \mathrm{C}, 2.1 \% \mathrm{O}, 1.0 \% \mathrm{~S}$ and $0.8 \% \mathrm{~N}$, but both layers exhibit higher $\mathrm{S}$ and $\mathrm{N}$ content. From Table 3 , it is found that the relative \% of PCPDTBT at 0 hours is higher for both films if the polymer and fullerene content was homogenously distributed; one should expect $25 \%$ relative PCPDTBT if this was the case. However, the measured concentration of PCPDTBT is $58 \%$ for films with ODT and $48 \%$ for films with CIN. This indicates that both layers exhibit an enrichment of PCPDTBT at the surface; however, a greater enrichment occurs at the surface for blends with ODT. Cl was not detected in the sample produced using CIN. This suggests that either the CIN is evaporated when the samples were transferred into a UHV environment or that it is present in lower concentration than the typical detection limit for XPS

On ageing, both samples shows an expected trend i.e. much higher $\mathrm{O}$, higher $\mathrm{N}$, lower $\mathrm{S}$ and correspondingly lower $\mathrm{C}$. Carbon appears as an oxygen-containing hydrocarbon with little evidence of aromatic species (the curve fitting of this data is not shown). Oxygen increases in intensity and shows two broad components attributable to $\mathrm{C}=\mathrm{O}$ and $\mathrm{C}-\mathrm{O}$ with the $\mathrm{C}=\mathrm{O}$ greater in relative proportion. Table 3 shows the peak area ratios of the $S 2 p$ and $C 1$ s peaks for the top surfaces. The changes in concentration of oxygen between the samples made with ODT and CIN is relatively small. However, subtle differences can be observed in the individual spectra.

The curve-fitted XPS data for the $S 2 p$ peaks is shown in figure 5 . The curve-fits are constrained to a physical model whereby the all $S 2 p_{3 / 2}-2 p_{1 / 2}$ doublet separations are fixed at 1.13 $\mathrm{eV}$, and all $S 2 p_{3 / 2}-2 p_{1 / 2}$ doublet intensity ratios are fixed to the ratios of the calculated HartreeSlater cross sections for photoemission from these levels at the Al K-alpha X-ray energy [14]. Based upon this physical model, the \% of $S$ in an oxidised state can be calculated. This gives $43.5 \%$ of the $S$ in the oxidised state for the sample prepared with ODT, and $30.86 \%$ of the $S$ in the oxidised state for the sample prepared with $\mathrm{Cl}-\mathrm{N}$. It is clear that the S component of the PCPDTDT in the sample made with ODT shows a greater susceptibility to oxidation than the one prepared with $\mathrm{CIN}$. This indicates samples made with ODT are more susceptible to oxidation, possibly due to the increased enrichment of PCPDTBT at the air facing surface, which is confirmed by the relative $\%$ of PCPDTBT at the surface data in table 3. The level of oxidised $\mathrm{S}$ is increased after light soaking when using ODT, however the magnitude of this effect does not correlate to the loss of performance observed between the two cells in working OPV cells. It can, therefore, be concluded that processing additives impair the performance of OPV cells via a combination of chemical and physical changes. 


\section{Conclusion}

PCPDTBT:PC ${ }_{71} B M$ solar cells are normally made with the inclusion of the processing additive, ODT, which is known to lead to enhanced initial PCE, due to improved morphology. However this additive is known to increase the degradation rate, so this paper has investigated the selection of alternative napthalene-based processing additives in order to improve the stability. By using a less volatile processing additive such as $\mathrm{BrN}$ or $\mathrm{CIN}$, a greater surface roughness is observed with aggregation observed from AFM topographic scans. Such morphology leads to lower initial PCE due to the worse active layer morphology. However, the stability by using these additives is shown to be enhanced by a factor three, though the initial PCE is reduced as a result. By considering this trade-off between initial PCE and stability, it has been shown that the output power generated in the first 1000 hours is about a factor two greater.

\section{$\underline{\text { Acknowledgements }}$}

HW would like to thank Bangor University for his 125 scholarship enabling his PhD study. JK would like to thank Sêr Cymru national research network in Advanced Engineering and Materials and the Newton Research Collaboration Programme scheme through the Royal Academy for Engineering.

\section{$\underline{\text { References }}$}

[1] Waters, Huw, Noel Bristow, Omar Moudam, Shu-Wei Chang, Chun-Jen Su, Wei-Ru Wu, U-Ser Jeng, Masaki Horie, and Jeff Kettle. "Effect of processing additive 1, 8-octanedithiol on the lifetime of PCPDTBT based Organic Photovoltaics." Organic Electronics 15, no. 10 (2014): 2433-2438.

[2] Zawacka, Natalia K., Thomas R. Andersen, Jens W. Andreasen, Lea H. Rossander, Henrik F. Dam, Mikkel Jørgensen, and Frederik C. Krebs. "The influence of additives on the morphology and stability of roll-to-roll processed polymer solar cells studied through ex situ and in situ X-ray scattering." Journal of Materials Chemistry A 2, no. 43 (2014): 18644-18654.

[3] Kesters, Jurgen, Pieter Verstappen, Jorne Raymakers, Wouter Vanormelingen, Jeroen Drijkoningen, Jan D'Haen, Jean Manca, Laurence Lutsen, Dirk Vanderzande, and Wouter Maes. "Enhanced Organic Solar Cell Stability by Polymer (PCPDTBT) Side Chain Functionalization." Chemistry of Materials 27, no. 4 (2015): 1332-1341. 
[4] Lee, Jae Kwan, Wan Li Ma, Christoph J. Brabec, Jonathan Yuen, Ji Sun Moon, Jin Young Kim, Kwanghee Lee, Guillermo C. Bazan, and Alan J. Heeger. "Processing additives for improved efficiency from bulk heterojunction solar cells." Journal of the American Chemical Society 130, no. 11 (2008): 3619-3623.

[5] Peet, Jeffery, Jin Young Kim, Nelson E. Coates, Wang Li Ma, Daniel Moses, Alan J. Heeger, and Guillermo C. Bazan. "Efficiency enhancement in low-bandgap polymer solar cells by processing with alkane dithiols." Nature materials 6, no. 7 (2007): 497-500.

[6] Lou, Sylvia J., Jodi M. Szarko, Tao Xu, Luping Yu, Tobin J. Marks, and Lin X. Chen. "Effects of additives on the morphology of solution phase aggregates formed by active layer components of high-efficiency organic solar cells." Journal of the American Chemical Society 133, no. 51 (2011): 20661-20663.

[7] Lee, Jae Kwan, Wan Li Ma, Christoph J. Brabec, Jonathan Yuen, Ji Sun Moon, Jin Young Kim, Kwanghee Lee, Guillermo C. Bazan, and Alan J. Heeger. "Processing additives for improved efficiency from bulk heterojunction solar cells." Journal of the American Chemical Society 130, no. 11 (2008): 3619-3623.

[8] Liu, Xueliang, Sven Huettner, Zhuxia Rong, Michael Sommer, and Richard H. Friend. "Solvent additive control of morphology and crystallization in semiconducting polymer blends." Advanced Materials 24, no. 5 (2012): 669-674.

[9] Albrecht, Steve, Wolfram Schindler, Jona Kurpiers, Juliane Kniepert, James C. Blakesley, Ines Dumsch, Sybille Allard, Konstantinos Fostiropoulos, Ullrich Scherf, and Dieter Neher. "On the field dependence of free charge carrier generation and recombination in blends of PCPDTBT/PC70BM: Influence of solvent additives." The Journal of Physical Chemistry Letters 3, no. 5 (2012): 640-645.

[10] Taken from Sigma Aldrich website (http://www.sigmaaldrich.com/)

[11] Reese, Matthew O., Suren A. Gevorgyan, Mikkel Jørgensen, Eva Bundgaard, Sarah R. Kurtz, David S. Ginley, Dana C. Olson et al. "Consensus stability testing protocols for organic photovoltaic materials and devices." Solar Energy Materials and Solar Cells 95, no. 5 (2011): 1253-1267.

[12] Kettle, J., M. Horie, L. A. Majewski, B. R. Saunders, S. Tuladhar, J. Nelson, and M. L. Turner. "Optimisation of PCPDTBT solar cells using polymer synthesis with Suzuki coupling." Solar Energy Materials and Solar Cells 95, no. 8 (2011): 2186-2193. 
[13] Boland, Patrick, Keejoo Lee, and Gon Namkoong. "Device optimization in PCPDTBT: PCBM plastic solar cells." Solar Energy Materials and Solar Cells 94, no. 5 (2010): 915-920.

[14] Scofield. J. H. "Hartree-Slater subshell photoionization cross-sections at 1254 and 1487 eV." Journal of Electron Spectroscopy and Related Phenomena 8, no 2 (1976): 129-137

Table 1: Mean and standard deviation value for PCPDTBT.PC ${ }_{71} B M$ based cells cast with 1chloronaphthalene $(\mathrm{CIN})$ and 1-bromonaphthalene $(\mathrm{BrN})$ additives respectively. PCPDTBT.PC ${ }_{71} \mathrm{BM}$ cast with ODT was included as a control. Characterised under AM1.5G conditions $\left(100 \mathrm{~mW} . \mathrm{cm}^{-2}\right)$.

\begin{tabular}{lrrr}
\hline & CIN & BrN & ODT \\
\hline $\mathrm{V}_{\mathrm{OCC}}(\mathrm{V})$ & $0.64 \pm 0.01$ & $0.63 \pm 0.01$ & $0.62 \pm 0.00$ \\
$\mathrm{~J}_{\mathrm{SC}}\left(\mathrm{mA} . \mathrm{cm}^{-2}\right)$ & $-7.71 \pm 0.29$ & $-7.20 \pm 0.28$ & $-9.05 \pm 0.38$ \\
FF (\%) & $46.53 \pm 1.04$ & $38.00 \pm 2.78$ & $51.05 \pm 0.88$ \\
PCE (\%) & $2.30 \pm 0.07$ & $1.72 \pm 0.20$ & $2.95 \pm 0.10$ \\
Boiling point & & & \\
\hline
\end{tabular}

Table 2: The sum of the electrical power generated over the first 1000 hours of PCPDTBT.PC ${ }_{71} B M$ cells, made with 1-chloronaphthalene, 1-bromonaphthalene and ODT respectively. Also included are the approximate times until the OPV performance is $50 \%$ of the original value $\left(T_{50 \%}\right)$, and additive boiling temperatures (B.T.).

\begin{tabular}{|c|c|c|c|}
\hline Additive & $\begin{array}{c}\text { Electrical Power } \\
\left(\mathrm{mWh} . \mathrm{cm}^{-2}\right) \\
{\left[\mathrm{T}_{100 \%}-\mathrm{T}_{10 \%}\right]}\end{array}$ & $\begin{array}{c}\text { Time to reach } 50 \% \text { of } \\
\text { initial PCE }\left(\mathrm{T}_{50 \%}\right) \\
\text { (hours) }\end{array}$ & B.T. (ㄷ) \\
\hline 1-bromonaphthalene & 260.58 & 121.47 & 119 \\
\hline 1-chloronaphthalene & 292.56 & 88.63 & 139 \\
\hline ODT & 130.93 & 24.75 & 270 \\
\hline
\end{tabular}


Table 3: The relative atomic concentrations in PCPDTBT:PC71BM blends at 0 and $300 \mathrm{~h}$ when blended with the processing additives 1,8-octanedithiol (ODT) or 1-chloronaphthalene (CIN) w/w of $2.5 \%$

\begin{tabular}{|c|c|c|c|c|}
\hline \multirow[b]{3}{*}{ Name } & \multicolumn{4}{|c|}{ Atom $\%$ concentration } \\
\hline & \multicolumn{2}{|c|}{ PCPDTBT:PC ${ }_{71} \mathrm{BM}$ with CIN } & \multicolumn{2}{|c|}{ PCPDTBT:PC ${ }_{71}$ BM with ODT } \\
\hline & O hours & 300 hours & O hours & 300 hours \\
\hline $\mathrm{C} 1 \mathrm{~s}$ & 91.50 & 71.88 & 90.84 & 69.48 \\
\hline 0 1s & 1.61 & 22.58 & 1.26 & 23.48 \\
\hline $\mathrm{N} 1 \mathrm{~s}$ & 2.41 & 2.90 & 2.56 & 3.73 \\
\hline$S 2 p$ & 3.39 & 2.64 & 5.34 & 3.31 \\
\hline$C: S$ & 20.38 & 27.23 & 17.01 & 20.99 \\
\hline $\begin{array}{l}\text { Relative \% of } \\
\text { PCPDTBT }\end{array}$ & 48.0 & 42.1 & 58.0 & 52.8 \\
\hline
\end{tabular}




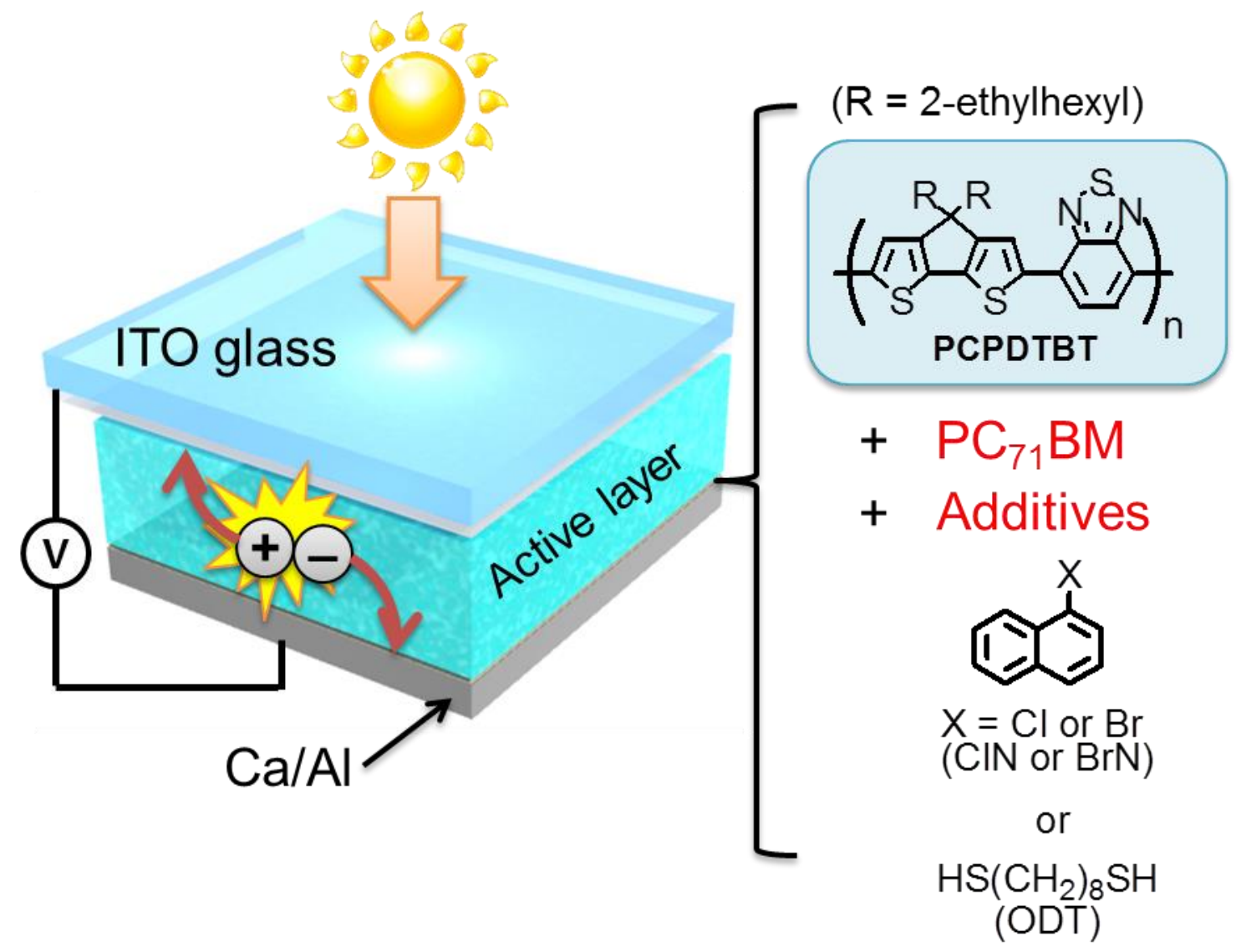

Figure 1: Schematic of device architecture used for these tests consisting of and ITO electrode, PEDOT:PSS hole transport layer, active layer and calcium/Aluminium cathode.

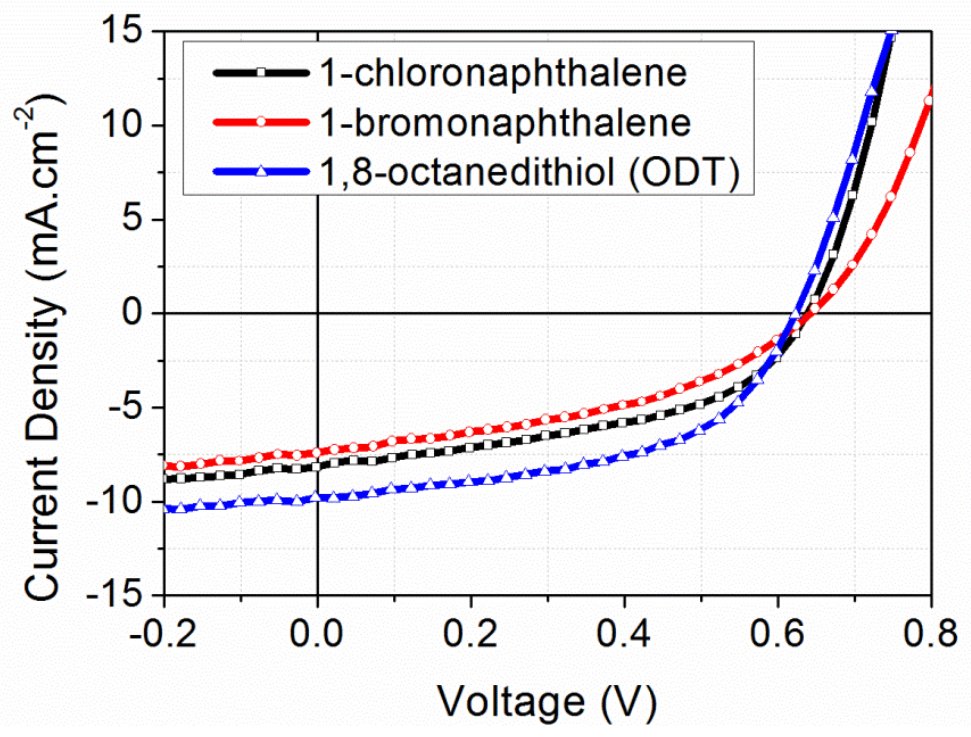


Figure 2: IV characteristics of the PCPDTBT:PC71BM solar cells made with ODT, $\mathrm{CIN}$ and $\mathrm{BrN}$ additives and tested under Am1.5G irradiation

(a)

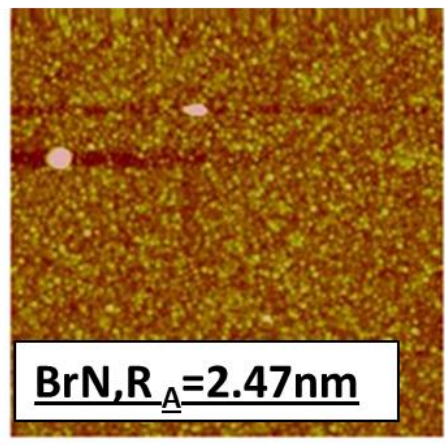

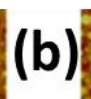

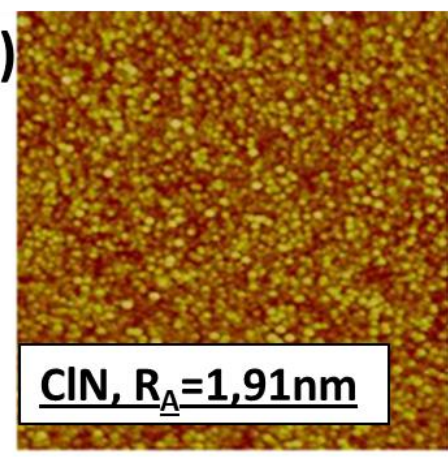

(c)

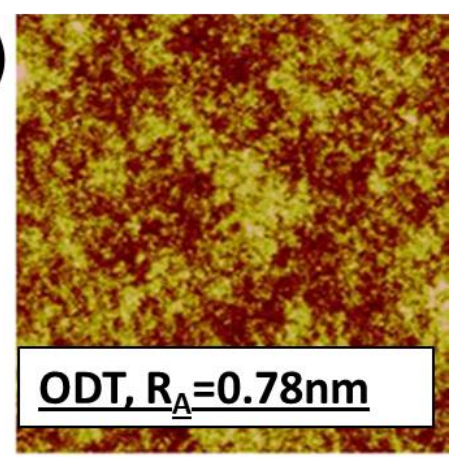

Figure 3: Topographic scans $(5 \mu \mathrm{m} \times 5 \mu \mathrm{m})$ using AFM for films of PCPDTBT.PC71BM with (a) 1bromonaphthalene, (b) 1-chloronaphthalene or (c) ODT (c)

(a)

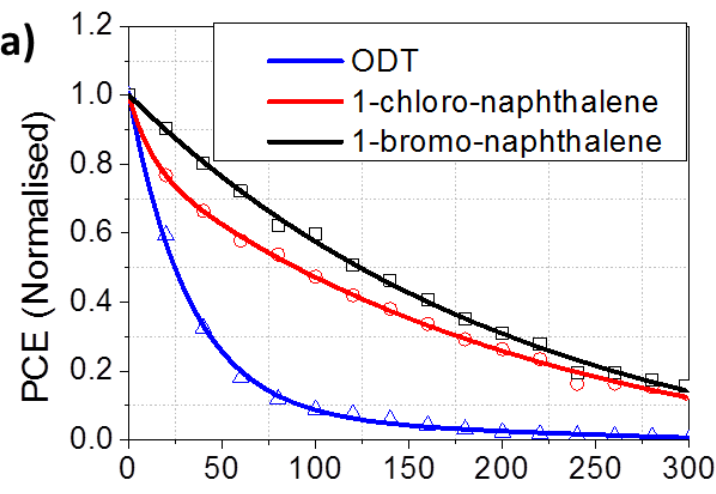

(c)

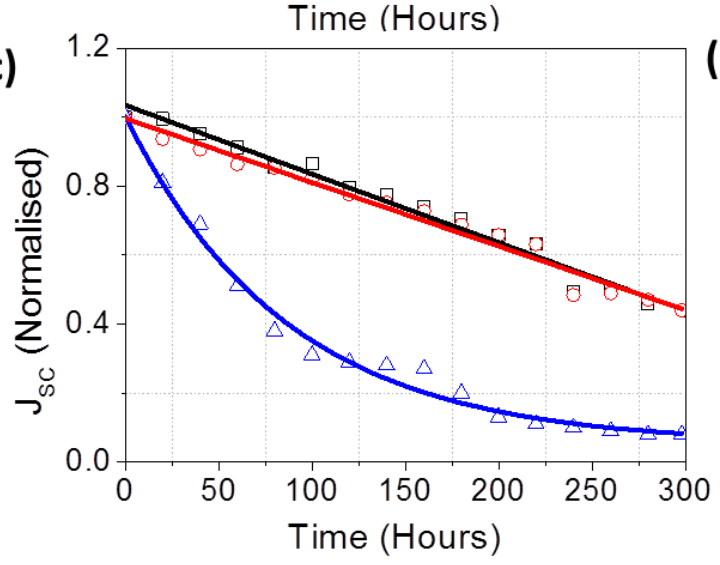

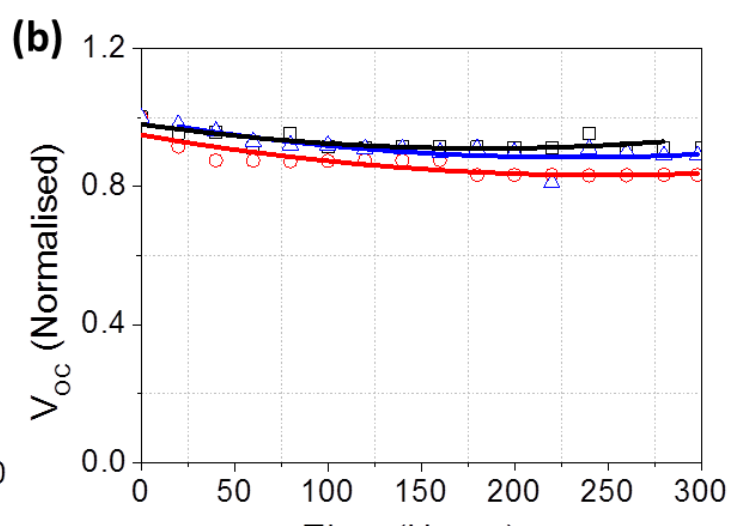

(d)

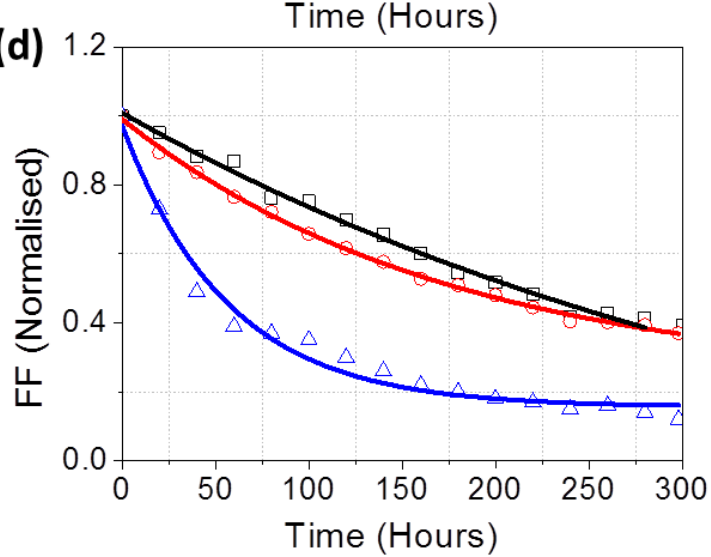

Figure 4: OPV cell characteristics for $\mathrm{PCPDTBT}: \mathrm{PC}_{71} \mathrm{BM}$ made with $\mathrm{CIN}$ or $\mathrm{BrN}$ processing additives. Shown are the variations of the $\mathrm{PCE}, \mathrm{V}_{\mathrm{OC}}, \mathrm{J}_{\mathrm{SC}}$, and FF with time, all normalised from their starting values. 

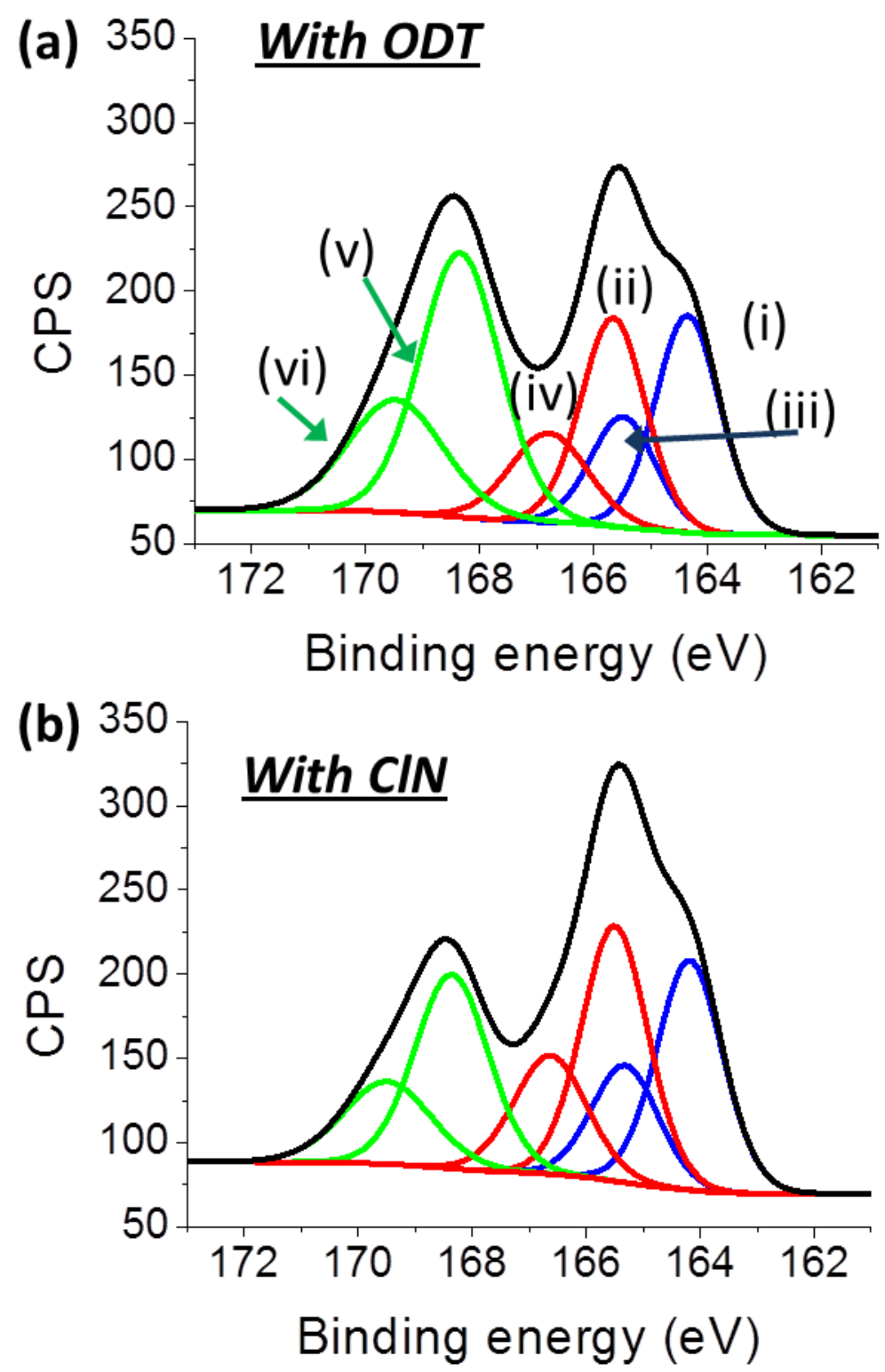

Figure 5: XPS spectra and curve fitting of PCPDTBT:PC ${ }_{71} B M$ OPVs made with (a) ODT additive and (b) $\mathrm{CIN}$ additive and aged for $300 \mathrm{~h}$ using ambient air light soaking facilities for the S2p peak only. In figure (a) only, the curved fitted positions of the (i) thiophene $S 2 p 3 / 2$ (ii) thiadiazole $S 2 p 3 / 2$, (iii) thiophene $S 2 p 1 / 2$, (iv) thiadiazole $S 2 p 1 / 2$, (v) oxidised $S 2 p 3 / 2$ and (vi) oxidised $S 2 p 1 / 2$ peaks are shown. 